\title{
A New Joint Denoising Algorithm for High-G Calibration of MEMS Accelerometer Based on VMD-PE-Wavelet Threshold
}

\author{
Huiliang Cao $\mathbb{D}^{1},{ }^{1}$ Zekai Zhang $\mathbb{D}^{1},{ }^{1}$ Yu Zheng, ${ }^{2}$ Hao Guo, ${ }^{1}$ Rui Zhao $\mathbb{D}^{1},{ }^{1}$ Yunbo Shi $\mathbb{D}^{1}$, \\ and Xiujian Chou ${ }^{1}$ \\ ${ }^{1}$ School of Instrument and Electronics, North University of China, Taiyuan 030051, China
${ }^{2}$ East China Institute of Photo-electron IC, Bengbu 233000, China \\ Correspondence should be addressed to Zekai Zhang; 1706004544@st.nuc.edu.cn and Yunbo Shi; shiyunbo@nuc.edu.cn
}

Received 4 May 2020; Revised 19 November 2020; Accepted 1 December 2020; Published 18 January 2021

Academic Editor: Arturo García Garcia-Perez

Copyright (C) 2021 Huiliang Cao et al. This is an open access article distributed under the Creative Commons Attribution License, which permits unrestricted use, distribution, and reproduction in any medium, provided the original work is properly cited.

Recently, the High-G MEMS accelerometer (HGMA) has been used in navigation, mechanical property detection, consumer electronics, and other fields widely. As the core component of a measuring system, it is very crucial to enhance the calibration accuracy of the accelerometer. In order to remove the noises in the accelerometer output signals to enhance its calibration accuracy, a combined denoising method which combines variational mode decomposition (VMD) with permutation entropy (PE) and wavelet threshold is given in this article. For the sake of overcoming the defect of signal distortion caused by the traditional denoising methods, this joint denoising method combines the good decomposition characteristics of VMD and the good denoising ability of wavelet threshold and introduces PE as a judgment criterion to achieve a good balance between denoising effect and signal fidelity. The combination of PE and VMD not only avoids the phenomenon of mode aliasing but also improves the ability to identify the noise components, which makes the wavelet threshold denoising more specific. Firstly, some intrinsic mode functions (IMFs) are obtained by using VMD to decompose the complex signal containing noise which is outputted from the accelerometer. Secondly, the IMF components can be divided into noise IMF components, mixed IMF components, and useful IMF components by PE algorithm. Thirdly, the noise IMF components can be discarded directly, and then the mixed IMF components can be denoised by wavelet threshold to obtain the noiseless IMF components; in addition, the useful IMF components need to be retained. Finally, the final denoising signal can be obtained by reconstructing the IMF components which have been denoised by the wavelet threshold and the useful IMF components retained before denoising. The experimental results prove that the combined denoising algorithm combines the merits of VMD, PE, and wavelet threshold, and this new algorithm has a good performance in the calibration denoising of accelerometer. Compared with the serious signal distortion caused by using only EMD or wavelet threshold, this method not only has a good denoising effect (the noises in the static part are eliminated by 99.97\% and the SNR of the dynamic part is raised to 18.56) but also can maintain a good signal fidelity (the error of shock peak amplitude is $3.4 \%$, the error of vibration peak amplitude is $0.4 \%$, and the correlation coefficient between the denoising signals and dynamic part is as high as 0.982 ).

\section{Introduction}

With the rapid progress of MEMS technologies, the research on inertial sensor components has been developed well. The High-G MEMS accelerometer is an outstanding representative of the inertial sensors. It not only overcomes the disadvantages of the traditional sensors such as large volume and large weight but also has the features of the high efficiency, high reliability, and high sensitivity [1-3]. Based on these advantages, it has been used in missile guidance, navigation, consumer electronics, and other fields widely [4-6]. As the core component of a measuring system, the performance of HGMA largely determines the system precision. Inevitably, due to the influences of the sensor itself, the hardware circuit, and the working environment, the accelerometer output signals are often mixed with noises, so it is essential to adopt the denoising algorithm to remove the noises in the output signals to improve the accuracy of the accelerometer $[7,8]$. 
For some common denoising algorithms, such as Fourier transform, wavelet transform, and Kalman filter, these algorithms inevitably have different degree of defects. The Fourier transform can reflect the relationship between the time function and the spectrum function, and it can be well applied to the analysis and processing of stationary signals, but it lacks the time-domain positioning function: that is, it can only give the overall effect of the signals, which makes it impossible to reflect the essential characteristics of the signals at a certain time. Compared with stationary signals, the instantaneous frequencies of nonstationary signals are functions of time, so the Fourier transform is not appropriate for the nonstationary analysis. The Kalman filter adopts matrix operation, which makes it have long computation time and serious waveform distortion. Wavelet transform is known as the microscope for signal analysis and processing because of its good ability to capture the local features of signals in time-frequency domain. Wavelet transform shrinkage (thresholding) denoising algorithm which was first proposed by Donoho and Johnstone $[9,10]$ is often used to analyze the nonstationary signals. Although wavelet threshold has good denoising property, denoising the whole signal with wavelet threshold alone will cause signal distortion. With the emergence of new time-frequency analysis algorithms like the empirical mode decomposition (EMD) algorithm, local mean decomposition (LMD) algorithm, or some improved algorithms based on them, the nonstationary signal analysis techniques become more mature. EMD is an algorithm commonly used for nonstationary signal analysis, which was proposed by Dr. Norden Huang. EMD can decompose the complex signals into some IMFs on the basis of the time scale characteristics of the signals themselves. Once proposed, the algorithm has been adopted in seismic exploration, mechanical fault diagnosis, and other engineering fields widely. Similar to EMD, LMD is another adaptive decomposition algorithm proposed by Smith [11]. Its essence is to multiply two sets of functions (frequency modulation signals and the envelope signals) separated from given complex signals to get a series of physically meaningful product functions (PFs). However, both algorithms have different degree of defects. For example, EMD has the disadvantages of mode aliasing and unreliable theoretical basis. Like EMD, LMD still has some disadvantages such as mode aliasing and endpoint effect. Different from EMD and LMD, VMD proposed by Dragomiretskiy et al. [12] achieves mode decomposition through seeking the optimal solutions in variational model, which overcomes the shortcomings of mode aliasing and has a solid theoretical basis. VMD is widely applied to related engineering fields [13-16] due to its separation characteristics and noise robustness.

Although the classical denoising methods proposed above have been widely used in the engineering fields, they all have different degree of defects. Recently, more and more experimental results show that hybrid denoising algorithms have better performance than single denoising methods [17-24]. Due to the good denoising characteristics of wavelet threshold, it is usually used in combination with EMD to denoise ECG signals and diagnose mechanical faults [22, 23].
With the introduction of VMD, this algorithm not only overcomes the inherent shortcomings of EMD and LMD such as mode aliasing and endpoint effect but also has many merits such as good noise robustness and solid theoretical foundation, so it has been widely used since it was proposed. Lahmiri et al. compared the denoising effect of the combination of VMD and wavelet threshold and the denoising effect of EMD and wavelet threshold on the ECG signal and concluded that the denoising method combined with VMD and wavelet threshold is more effective [20]. In these similar joint denoising methods, VMD is often used for signal decomposition. Although VMD algorithm has good decomposition effect, it also has the problem of over-decomposition. In addition, the wavelet threshold is used to denoise the whole signal or denoise each mode function obtained by the VMD, and then the final denoising signal is obtained by signal reconstruction. However, this often leads to signal distortion in the denoising process. Moreover, $\mathrm{PE}$ was proposed by Bandt and Pompe [25], which is adopted to measure the degree of random and dynamic mutation of time series or complex signals. It is characterized by simple calculation, good detection effect, and strong antinoise ability. In recent years, many scholars have combined PE with other algorithms to analyze nonstationary signals [26-29].

In this article, in order to remove noises to enhance the accuracy of the accelerometer, VMD is combined with the wavelet threshold; in addition, $\mathrm{PE}$ is introduced as the judgment criterion, finally forming the VMD-PE-wavelet threshold combined denoising method. The principle of this joint denoising method is as follows: firstly, a series of IMFs are obtained through using VMD to decompose the HGMA output signals. Then, these IMF components can be divided into noise IMF components, mixed IMF components, and useful IMF components by PE. The noise IMF component can be discarded directly, and then the mixed IMF components can be denoised by wavelet threshold to obtain the noiseless IMF components; in addition, the useful IMF components need to be retained. Finally, the final denoising signal can be obtained by reconstructing the IMF components which have been denoised by the wavelet threshold and useful IMF components retained before denoising. The innovation of this joint denoising method lies in the introduction of permutation entropy to classify the mode functions obtained after the signal decomposition. The combination of PE and VMD not only can avoid mode aliasing but also has good recognition ability, making the denoising more targeted. Compared with the denoising methods which only use EMD or wavelet threshold, the proposed method achieves a balance between denoising effect and signal fidelity.

In addition, we have improved the original experimental analysis method. The output signal of HGMA can be divided into two parts: static phase and dynamic phase. As the amplitude and frequency characteristics of these two phases are different, it is not appropriate to carry out denoising analysis on the whole signal. Better results can be obtained by dividing the output signal into static phase and dynamic phase and denoising them, respectively. The dynamic part 
can be subdivided into the shock stage and the vibration stage, and the static part is also called the preparation stage. This article compares the performance of different denoising methods in these three stages from two aspects: time domain and frequency domain. The experimental results prove that the combined denoising algorithm introduced in this article has a good balance between denoising effect and signal retention. The static part is analyzed by Allan deviation, and the denoising signal noise is reduced by $99.97 \%$. Different denoising algorithms have the largest difference in the denoising effect in the dynamic part. Compared with the severe amplitude attenuation in the timefrequency domain caused by EMD or wavelet denoising, VMDPE-wavelet threshold not only has a good denoising effect (the SNR of the dynamic part is raised to 18.56) but also can maintain a good signal fidelity (the error of shock peak amplitude is $3.4 \%$, the error of vibration peak amplitude is $0.4 \%$, and the correlation coefficient between the denoising signal and the dynamic part is as high as 0.982).

In order to eliminate the noises in the accelerometer output signals to enhance the calibration accuracy, this paper proposes a combined denoising algorithm (VMD-PEwavelet threshold) based on VMD and wavelet threshold and introduces permutation entropy (PE) as the judgment criterion. The experimental results prove that the combined denoising method has a good balance between denoising effect and signal fidelity. The specific content arrangement of this article is as follows. In Section 2, the VMD-PE-wavelet threshold algorithm is described in detail. Relevant structure and parameters of the accelerometer are described in Section 3. The experimental results and comparative analysis of different denoising algorithms are given in Section 4. Section 5 gives the conclusion.

\section{Algorithm}

2.1. The Introduction of Variational Mode Decomposition (VMD). Similar to EMD, VMD also decomposes the complex signals into intrinsic mode functions to implement signal decomposition. Different from the way that EMD obtains IMF components through cyclic screening, VMD obtains IMF components through iteratively seeking for the optimal solutions of the variational models. VMD can overcome some inherent defects of existing decomposition models (EMD and LMD), such as sensitivity to noise and sampling, mode aliasing, and endpoint effect. VMD can extract modes simultaneously by constructing a nonrecursive variational mode decomposition model, which makes it have good performance. For a given complex input signal, its constraint variational model can be constructed as [12]

$$
\begin{aligned}
& \min _{\left\{u_{k}\right\}\left\{\omega_{k}\right\}}\left\{\sum_{k}\left\|\partial_{t}\left[\left[\delta(t)+\frac{j}{\pi t}\right] * u_{k}(t)\right] e^{-j \omega_{k} t}\right\|_{2}^{2}\right\}, \\
& \text { s.t. } \sum_{k} u_{k}=y(t),
\end{aligned}
$$

where $\left\{u_{k}\right\}=\left\{u_{1}, \ldots, u_{k}\right\}$ is a series of intrinsic mode functions (IMFs) obtained by the decomposition of signa $y(t)$, $\left\{w_{k}\right\}=\left\{w_{1}, \ldots, w_{k}\right\}$ is a set of central frequencies which belong to each intrinsic mode function, $\|\bullet\|_{2}^{2}$ is the Euclid norm, $\delta$ is the Dirac distribution, and $*$ is the convolution operator.

The augmented Lagrangian expressions can be obtained after inserting the quadratic penalty term factor $\alpha$ and the Lagrange multiplication operator $\lambda(t)$, which is

$$
\begin{aligned}
L\left(\left\{u_{k}\right\},\left\{\omega_{k}\right\}, \lambda\right)= & \alpha \sum_{k}\left\|\partial_{t}\left[\left(\delta(t)+\frac{j}{\pi t}\right) * u_{k}(t)\right] e^{-j \omega_{k} t}\right\|_{2}^{2} \\
& +\left\|y(t)-\sum_{k} u_{k}(t)\right\|_{2}^{2} \\
& +\left\langle\lambda(t), y(t)-\sum_{k} u_{k}(t)\right\rangle .
\end{aligned}
$$

The saddle point of the augmented Lagrangian function is sought iteratively by the alternating direction multiplier method (ADMM), that is, the above constrained variational model's optimal solution has been found, and all the intrinsic mode functions $u_{k}$ are

$$
\widehat{u}_{k}^{n+1}(\omega)=\frac{\widehat{y}(\omega)-\sum_{i>k} \widehat{u}_{i}(\omega)+(\widehat{\lambda}(\omega) t / 2)}{1+2 \alpha\left(\omega-\omega_{k}\right)^{2}} .
$$

The iteration process of $w_{k}$ and $\lambda(t)$ are described as formulas (4) and (5), respectively:

$$
\begin{aligned}
\widehat{\omega}_{k}^{n+1} & =\frac{\int_{0}^{\infty} \omega\left|\widehat{u}_{k}(\omega)\right|^{2} \mathrm{~d} \omega}{\int_{0}^{\infty}\left|\widehat{u}_{k}(\omega)\right|^{2} \mathrm{~d} \omega}, \\
\hat{\lambda}^{n+1}(\omega) & =\widehat{\lambda}^{n}(\omega)+\tau\left(\hat{y}(\omega) t-n \sum_{k} \widehat{u}_{k}^{n+1}(\omega)\right),
\end{aligned}
$$

where the symbol ${ }^{\wedge}$ represents the value update of the variable and $\tau$ is the update parameter.

The detailed steps of the variational modal decomposition method are shown in Figure 1.

2.2. The Introduction of Permutation Entropy (PE). PE is an algorithm commonly adopted to measure the degree of random and dynamic mutation of time series, which was proposed by Bandt and Pompe [25]. The unique advantages of this algorithm are as follows: first, the calculation is simple and the antinoise ability is strong; second, the algorithm is sensitive to time and can get higher resolution; and third, the output result of the algorithm has a better recognition of mutation information intuitively. When VMD is performed on the sensor output signals, a series of intrinsic mode functions (IMFs) are obtained. In order to achieve a balance between denoising effect and signal fidelity, in this article, PE is combined with VMD, and IMF components obtained after VMD can be classified by PE. The algorithm steps are [30] as follows.

2.2.1. Phase Space Reconstruction. Through phase space reconstruction, the original time series $\{Y(i), i=1,2,3, \ldots$, $\mathrm{n}$ \} is reconstructed into matrix $S$ : 


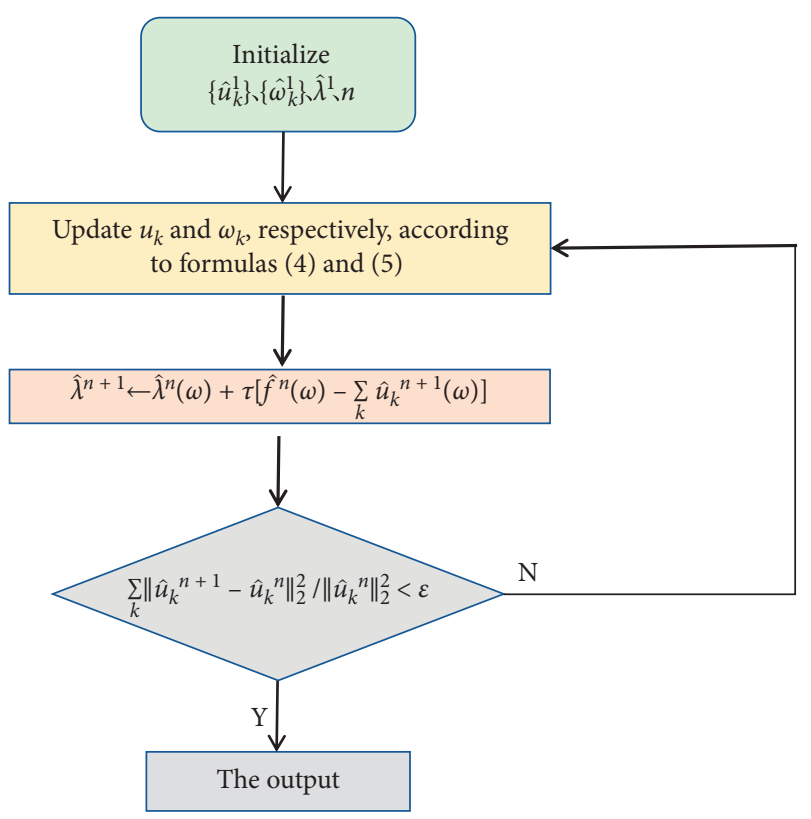

FIGURE 1: Specific steps of VMD algorithm.

$$
S=\left(\begin{array}{c}
s(1) \\
\vdots \\
s(j) \\
\vdots \\
s(k)
\end{array}\right)=\left(\begin{array}{cccc}
y(1) & y(1+\tau) & \cdots & y(1+(m-1) \tau) \\
\vdots & \vdots & & \vdots \\
y(j) & y(j+\tau) & \cdots & y(j+(m-1) \tau) \\
\vdots & \vdots & & \vdots \\
y(k) & y(k+\tau) & \cdots & y(k+(m-1) \tau)
\end{array}\right)
$$

where $m$ and $\tau$ are, respectively, two parameters of PE: embedded dimension and time delay, and each $S(j)$ is a reconstructed sequence.

2.2.2. Extraction of Symbol Sequence. Rearrange each vector in the reconstruction matrix numerically from small to large, and a new sequence can be obtained by rearranging the $J$ th component $S(j)=\{y(j), y(j+\tau), \ldots, y(j+(m-1) \tau)\}$ in the matrix $S$ :

$$
\begin{aligned}
& y\left(j+\left(i_{1}-1\right) \tau\right) \leq y\left(j+\left(i_{2}-1\right) \tau\right) \leq y\left(j+\left(i_{3}-1\right) \tau\right) \leq \cdots \\
& \quad \leq y\left(j+\left(i_{m-1}-1\right) \tau\right) \leq y\left(j+\left(i_{m}-1\right) \tau\right)
\end{aligned}
$$

where $i_{1}, i_{2}, \ldots, i_{m}$ represent the position of each element in this new sequence.

If there are equal values in the reconstructed component, which is

$$
y\left(j-\left(i_{p}-1\right) \tau\right)=y\left(j-\left(i_{q}-1\right) \tau\right),
$$

then, follow $i_{p}$ with $i_{q}$, that is to say, if $i_{p}<i_{q}$, then

$$
y\left(j-\left(i_{p}-1\right) \tau\right) \leq y\left(j-\left(i_{q}-1\right) \tau\right) \text {. }
$$

For any reconstructed sequence $S(j)=\left(i_{1}, i_{2}, i_{3}, \ldots, i_{m}\right)$, $j=1,2, \ldots, k$, since each sequence $S(j)$ has $m$ index symbols $\left(i_{1}, i_{2}, i_{3}, \ldots, i_{m}\right)$, there are $m$ ! symbol sequences, and the sequence $S(j)$ is just one of them, so $k \leq m$ !.
2.2.3. Calculation and Normalization of the Permutation Entropy. Each symbol sequence appears with certain probability. The probabilities corresponding to each symbol sequence can be denoted as $P_{1}, P_{2}, \ldots, P_{k}$. For any signal series $\{Y(j), i=1,2,3, \ldots, \mathrm{n}\}, k$ reconstruction vectors are obtained after the above two operations. The permutation entropy can be obtained by the form of Shannon entropy, which is

$$
P E(m)=-\sum_{j=1}^{j} p_{j} \ln p_{j} .
$$

When $p_{j}=1 / m$ !, then $P E(m)$ achieves its maximum ln $m$ !. For the sake of convenience, $P E(m)$ can be normalized by $\ln m !:$

$$
P E=\frac{P E(m)}{\ln m !} .
$$

After normalization, the value range of permutation entropy is limited to $[0,1]$. The value of PE reveals the complexity and the degree of random of the signals: the greater the permutation entropy, the more random the signals, and vice versa.

In addition, there are three key parameters in this algorithm: the length of signal $N$, the time delay factor $\tau$, and the embedded dimension $m$. For the embedded dimension $m, \mathrm{PE}$ algorithm effect will be affected when the value is too large, and when the value is too small, those reconstructed sequences could lose the original information. The value range of $m$ should be between 3-7, which was suggested by Bandt et al. [25, 31]. By analyzing the results obtained by using different $m$ values, this paper sets $m$ at 5 . Compared with the embedded dimension, the factor $\tau$ has little effect on the performance of $\mathrm{PE}$ algorithm. In this paper, $\tau$ is set at 1 . In addition, when the data length exceeds 1024, a stable calculation value can be obtained.

2.3. The Introduction of Wavelet Threshold Denoising. Wavelet threshold denoising is based on wavelet transform. After the wavelet decomposition, the wavelet coefficient generated by the signal is larger than that generated by the noise. According to this principle, a threshold value is introduced to process those wavelet coefficients, and then the final denoising signals can be obtained through reconstructing the processed wavelet coefficients. So, the wavelet threshold denoising is adopted to denoise the mixed IMF components in this article. The steps of the algorithm can be summarized as follows $[32,33]$.

2.3.1. Wavelet Decomposition. Before the wavelet decomposition, it is necessary to find the suitable wavelet base and decomposition layer for the decomposition signal, and different choices can get different denoising effects.

2.3.2. Thresholding of Wavelet Coefficients. The wavelet coefficients are classified by a given threshold value: those wavelet coefficients whose amplitude are greater than the 
given threshold value will be considered to be created by useful signals and need to be retained or shrunk appropriately. On the contrary, the wavelet coefficients will be considered to be created by the noises and need to be set to zero.

2.3.3. Wavelet Reconstruction. The final denoising signals can be gained through reconstructing those processed wavelet coefficients by inverse wavelet transform.

Different from noise, the signals have a certain continuity in the time domain, which is also reflected in the wavelet domain, that the amplitude of the wavelet coefficients of useful signals is greater than that of those wavelet coefficients generated by noises, so the noises and useful signals can be separated by the wavelet transform. Then, a threshold value should be determined when using the wavelet threshold denoising algorithm. When different threshold estimation and threshold function are selected, the effect of wavelet threshold denoising is also different [34], and there are many judgment criteria. Threshold selection methods are mainly divided into heuristic estimation (heursure), fixed threshold estimation (sqtwolog), extreme threshold estimation (minimaxi), unbiased likelihood estimation (rigsure), etc. Generally speaking, the first two threshold estimation methods (heursure and sqtwolog) are more decisive and easy to eliminate useful signals such as noise when denoising. However, the last threshold estimation method (rigsure) is relatively conservative and can keep the amplitude of the signal well, so this paper chooses the unbiased likelihood estimation method. The specific steps are as follows:

(1) Take the absolute value of each element in signal $y(j)$, reorder them in ascending order according to their absolute values, and then a new signal sequence $s(j)$ can be obtained:

$$
s(i)=(\operatorname{sort}(|y|))^{2}, \quad(i=0,1, \ldots, N-1) .
$$

(2) If the square root of the $J$ th element of $s(j)$ is chosen as the threshold value $(t h)$, then the risk generated by this threshold is denoted as rish $(j)$ :

$$
\begin{aligned}
\text { th } & =\sqrt{s(j)}, \quad(j=0,1, \ldots, N-1), \\
\operatorname{risk}(j) & =\frac{N-2 j+\sum_{i=1}^{j} s(i)+(N-j) s(N-j)}{N} .
\end{aligned}
$$

(3) The minimum risk point is found in the obtained risk curve rish $(j)$. The subscript of the element corresponding to the minimum risk point is $j_{\min }$, so the rigsure threshold is denoted as

$$
\text { th }=\sqrt{s\left(j_{\min }\right)}
$$

After determining the threshold, the next step is to choose the appropriate threshold function to filter the wavelet coefficients which contain the noise coefficients. Soft and hard threshold functions are two commonly used threshold functions. The similarity between the two methods is that when the wavelet coefficients are smaller than a given threshold value, these wavelet coefficients should be set to zero as noise coefficients. Differently, when the wavelet coefficients are larger than the given threshold value, hard threshold function will keep them unchanged, while the soft threshold function subtracts the threshold value from the wavelet coefficients, which makes the overall continuity of wavelet coefficients better, so this article chooses soft threshold function.

The hard threshold functions can be separately described as

$$
\eta(w, t h)= \begin{cases}w, & |w| \geq t h, \\ 0, & |w|<t h .\end{cases}
$$

The soft threshold function can be described as

$$
\eta(w, t h)= \begin{cases}\operatorname{sign}(w)(|w|-t h), & w \geq t h, \\ 0, & |w|<t h,\end{cases}
$$

where $w$ and th are wavelet coefficients and thresholds, respectively. $\lambda=\sigma \times \sqrt{2 \lg N}$, and the noise standard value is $\sigma=\operatorname{Median}|w| / 0.6745$, of which Median $|w|$ is the median of wavelet coefficients.

2.4. Introduction of VMD-PE-Wavelet Threshold. For the output signal of the accelerometer, the noise will often drown the useful signal. We hope to keep the useful signal to the maximum extent while removing the noise. With the development of nonlinear stationary signal denoising methods based on modal decomposition, signal denoising algorithms such as EMD and LMD emerge. The original signals can be decomposed into some mode functions from high to low frequency by these algorithms, and these algorithms achieve the purpose of denoising by directly discarding the high frequency components and reconstructing the remaining components. Although these methods have good noise reduction effect, they often lose useful signals. In order to achieve a balance between denoising effect and signal fidelity, in this article, VMD is combined with the wavelet threshold denoising and $\mathrm{PE}$ is introduced to classify the IMF components, which makes the wavelet threshold denoising more targeted. The steps of VMD-PE-wavelet threshold denoising algorithm are as follows.

2.4.1. Decomposing the Output Signals of Accelerometer with $V M D$. After the decomposition of the accelerometer output signal, a series of IMF components which contain useful component and noise component are obtained; therefore, these IMF components cannot be simply discarded as noise or retained as pure signal. So, PE is introduced here to distinguish these IMF components. 
2.4.2. Classifying the IMF Components Using PE. In this article, $\mathrm{PE}$ algorithm is introduced to improve the problem in distinguishing IMF components obtained after decomposition. Compared with the noise, the useful signal is more regular in time and the corresponding $\mathrm{PE}$ value is relatively small. In the VMD-PE-wavelet threshold denoising method, the IMF components are calculated to obtain their PE values, and then the IMF components are reordered according to their PE values. The IMF component with a small PE value is considered as the useful IMF component, the IMF component with a moderate $\mathrm{PE}$ value is considered as the mixed IMF component, which is composed of noise and useful signals, and the IMF component with a large PE value is considered as the noise IMF component.

2.4.3. Denoising Processing. The IMFs can be divided into noise IMF components, mixed IMF components, and useful IMF components by PE. The noise IMF components can be discarded directly, and then the mixed IMF components can be denoised by wavelet threshold to obtain the noiseless IMF components; in addition, the useful IMF components need to be retained.

2.4.4. Reconstruction of the Signal. In this article, the essence of signal reconstruction is discarding the noise IMF components and then reconstructing the mixed IMF components which have been denoised by the wavelet threshold with useful IMF components to obtain the final denoising signals.

The specific steps of VMD-PE-wavelet threshold are given in Figure 2.

\section{High-G MEMS Accelerometer}

The signals to be analyzed in this article come from a newly designed and manufactured HGMA. The accelerometer is known for its high impact survival rate and wide measurement ranges. HGMA conducts correlation measurement through piezoresistive effect, and the detection signal is output in the form of voltage.

3.1. Structure Description of the HGMA. As shown in Figure 3 , on the structure of HGMA, the four-beam island structure is adopted. To facilitate processing, the structures of the HGMA, such as the frame, the four beams, and the central mass, are made into rectangular shapes. Specific structural parameters are also given in Figure 3.

A coordinate system can be determined by using the cross section of the HGMA as a reference, where the $\mathrm{Z}$-axis is the direction along the central dividing line of cross section; in addition, its positive direction is downwards. X-axis is the direction along the other middle line, and the positive direction is to the right. The beam has three shape parameters: length, width, and thickness, which can be denoted as $a_{1}, b_{1}$, and $c_{1}$, respectively. Moreover, the shape parameters of the mass can be denoted as $a_{2}, b_{2}$, and $c_{2}$, and the sizes of these shape parameters are given in Table 1.
Four main resonance modes of the accelerometer are simulated by ANSYS soft. The simulation analysis of these four resonant modes of the accelerometer is, respectively, given in Figure 4. The accelerometer operates in its first mode, where the mass moves along $\mathrm{Z}$-axis. The second and third mode masses rotate along the $\mathrm{X}$-axis and $\mathrm{Y}$-axis, respectively. In addition, for the fourth resonant mode of the accelerometer, both the mass and frame move along $\mathrm{Z}$-axis. Their resonant frequencies are given in Table 2, indicating that the resonant frequency of the working mode (the first resonant mode) is $408 \mathrm{kHz}$, and the frequencies of the remaining three resonant modes are $667 \mathrm{kHz}, 671 \mathrm{kHz}$, and $1119 \mathrm{kHz}$, respectively. In particular, there is a gap of $260 \mathrm{kHz}$ between the second and first order modes, which indicates that there is a small coupling motion between the two resonant modes, which is conducive to the linearization of the HGMA. In addition, in Figure 5, the SEM and confocal microscope photos of HGMA structure are given.

\section{Experiment and Analysis}

4.1. Experiment. The experimental device is shown in Figure 6. From left to right, there are recycling box, deformable instrument, computer, Hopkinson bar, and compressed air, which together constitute the Hopkinson bar calibration system. In addition, the power supply provides $a+5 \mathrm{~V}$ voltage for HGMA, the temperature is kept at $25^{\circ} \mathrm{C}$ (room temperature), and the sampling points are 19243.

In detail, the schematic diagram and experimental apparatus for the dynamic linear incremental impact system, which adopts the developed dual-warhead Hopkinson bar, are given in Figure 6. As shown in Figure 6(a), the entire test system includes an accelerometer measurement part, a launch and impact part, the pressure control, and data acquisition parts. In addition, Figures 6(c) and 6(d) show the details of the launch tube and the measured accelerometer, respectively.

In Figure 6, the accelerometer is installed at the bottom of the base to detect the acceleration produced by the bullet hitting the Hopkinson bar, which ranges from $1.5 \times 10^{5} \mathrm{~g}$ to $2.0 \times 10^{5} \mathrm{~g}$. The grating is placed near the base to detect the Doppler shift caused by the acceleration. Pressure chambers 1 and 2 are used to generate pressures up to $1 \mathrm{MPa}$. In the experiment of inside bullet or outside bullet, the bullet hits Hopkinson bar and generates the longitudinal elastic compression wave on the other side of Hopkinson bar. The accelerometer installed on the side of the rod will fly out immediately under the action of a quasi-half-sine acceleration pulse from the compression wave. The Doppler shift can be measured by the grating which, installed on the base, measures according to the acceleration measured by the accelerometer. Then, the measured signals are converted into voltage signals and input into the OP amplifier, and finally the voltage signals are analyzed by the data acquisition part.

4.2. Analysis. The collected output signals in the experiment are shown in Figure 7. Generally speaking, the signal can be 


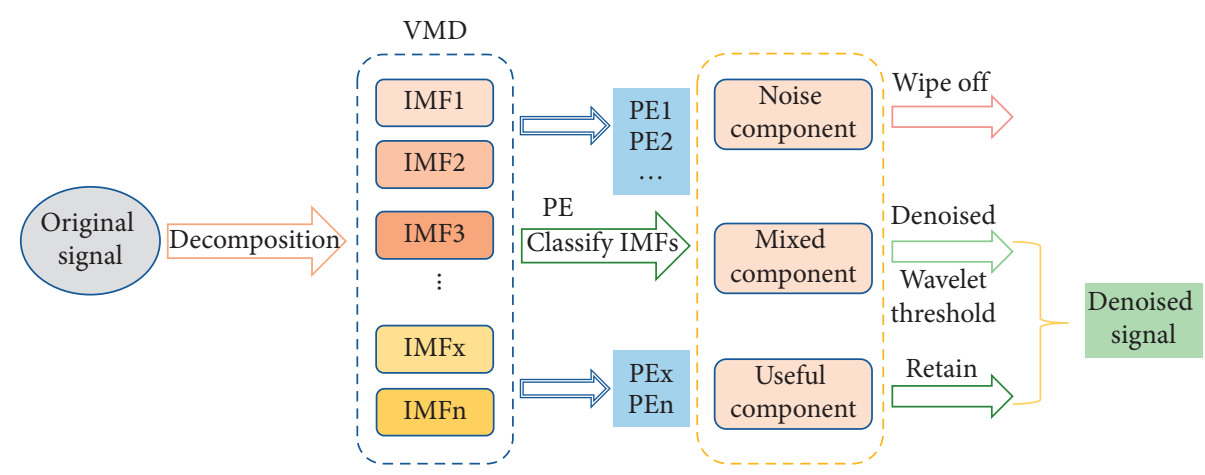

Figure 2: The steps of VMD-PE-wavelet threshold algorithm.
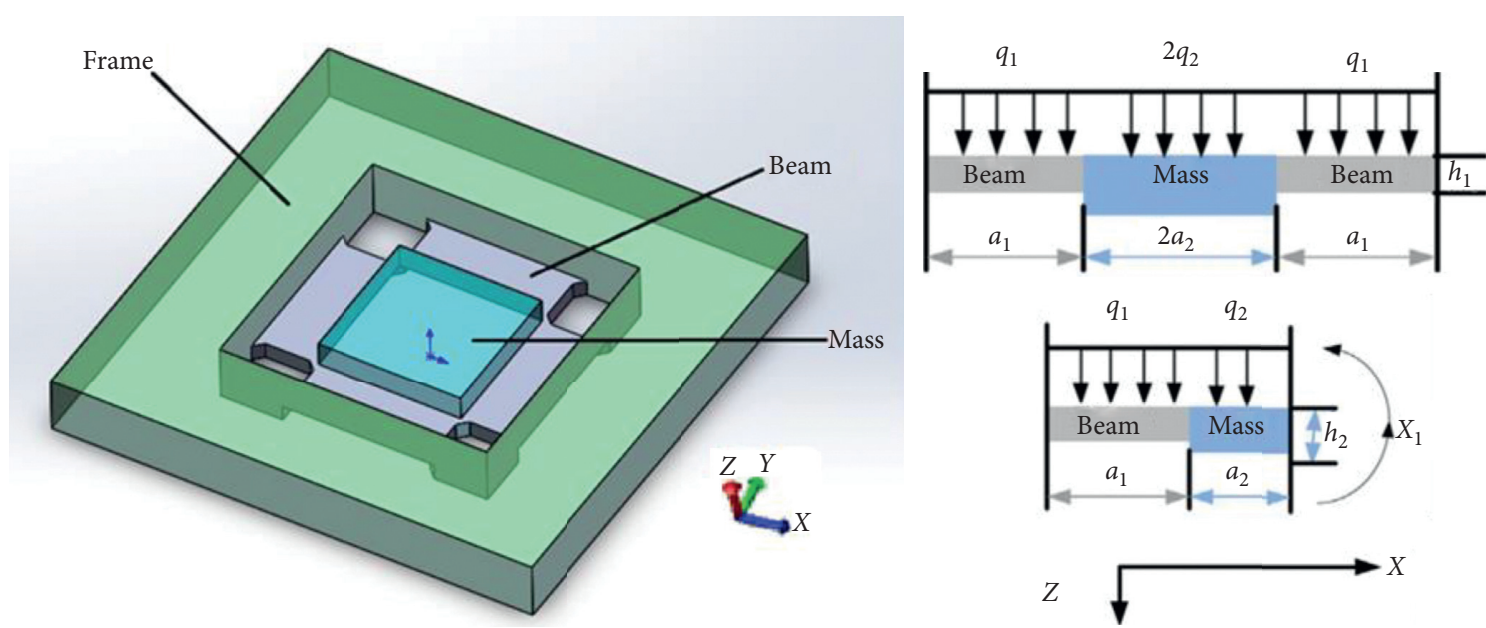

FIgURE 3: The structure schematic and size of HGMA.

TABLE 1: Structure parameters of the accelerometer's beam and mass.

\begin{tabular}{lcccccc}
\hline & & & & & The mass \\
Shape parameters & Length $\left(a_{1}\right)$ & Width $\left(b_{1}\right)$ & Height $\left(c_{1}\right)$ & Length $\left(a_{2}\right)$ & Width $\left(b_{2}\right)$ & Height $\left(c_{1}\right)$ \\
\hline Size $(\mu \mathrm{m})$ & 350 & 800 & 80 & 800 & 800 & 200 \\
\hline
\end{tabular}

divided into two parts: static signal (preparing stage) and dynamic signal. When there is no external interference, the accelerometer output is a static signal. In general, static signal contains a wealth of random noise. The statistical characteristics of noise in HGMA signal can be obtained from the static output signal. Moreover, the dynamic signal caused by external impact consists of two phases: the shock phase and the vibration phase. For this calibration experiment, the shock stage is the main part, and the vibration stage contains the vibration information which can reflect the dynamic characteristics of HGMA. Since the amplitude and frequency characteristics of the output signals in the static stage and the dynamic stage are different, the direct denoising output signal will affect the denoising effect. Therefore, this paper analyzes the static stage and the dynamic stage, respectively, and obtains a better denoising effect. Since the dynamic output signal of HGMA contains a large amount of vibration information, this paper mainly analyzes and demonstrates the denoising effects of different algorithms in the dynamic stage.

According to the VMD-PE-wavelet threshold denoising algorithm steps, firstly, the output signal is decomposed by VMD. Different from EMD, LMD, and other decomposition algorithms, before the VMD, relevant parameters need to be determined, such as the number of decomposition layers $K$ and quadratic penalty factor $\alpha$, among which, the determination of $K$ has the greatest impact on the decomposition effect. In this article, $K$ is determined according to the principle of center frequency proximity, that is, initial $K$ value is set first, then $K$ value is increased successively, VMD is conducted, and the center frequency of each mode is obtained. When two center frequencies are similar, it is judged to be over-decomposition, then the previous $K$ value is taken as the mode number, and the default value of the remaining parameters is adopted in this paper. With different $k$ values selected, the center frequency of each mode 

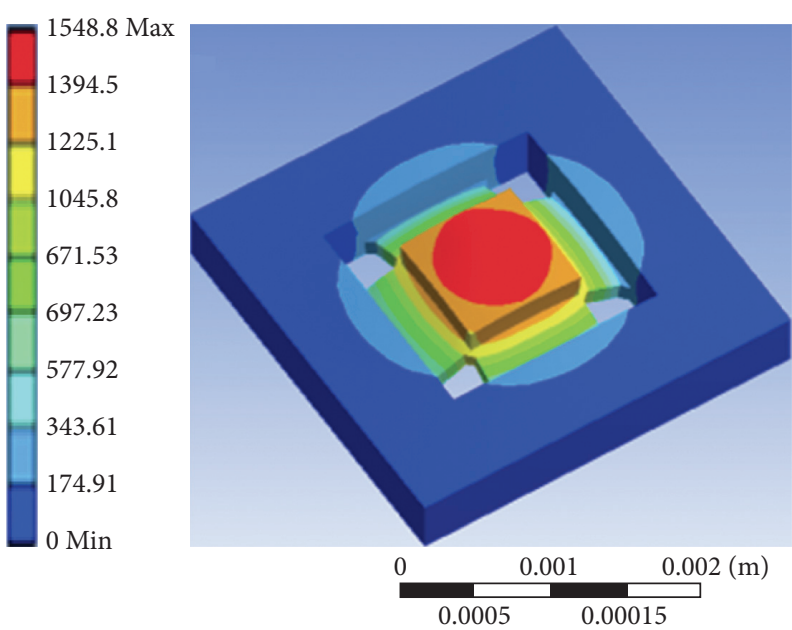

(a)
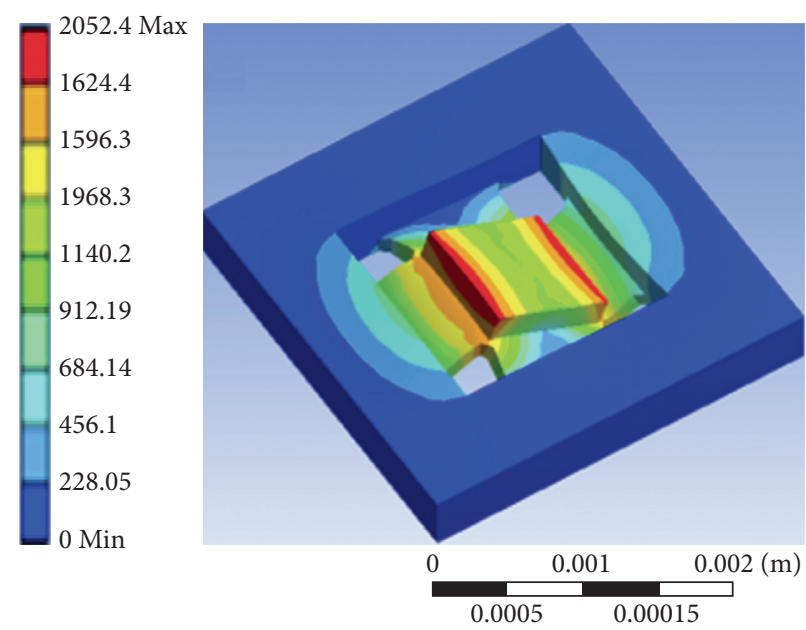

(c)

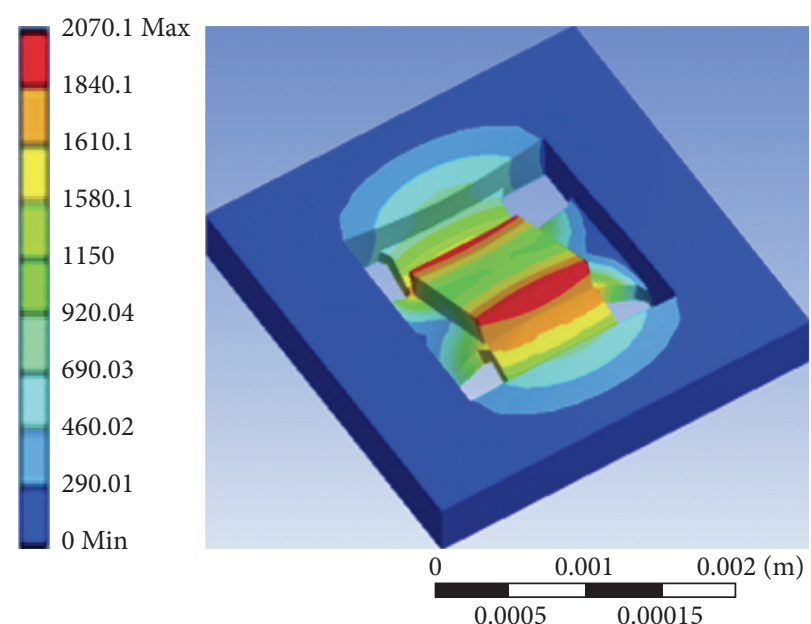

(b)
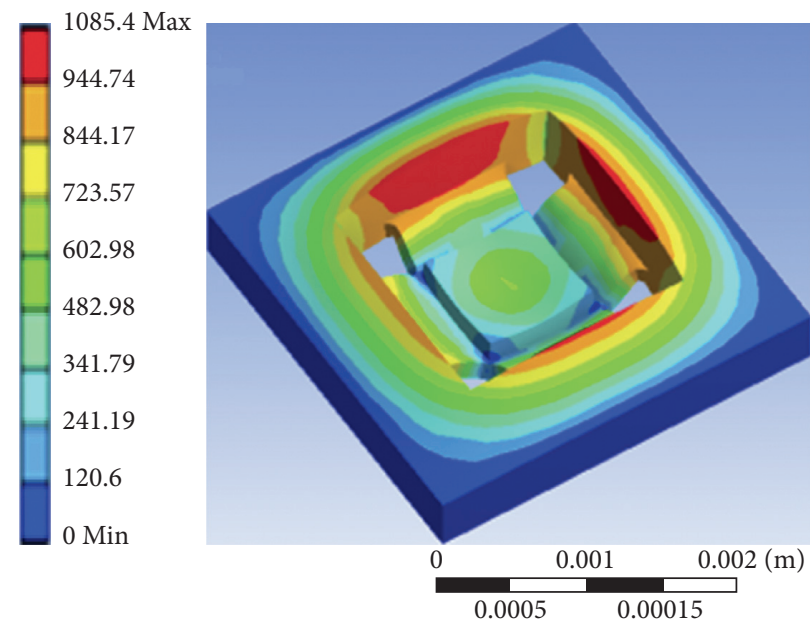

(d)

FIGURE 4: Simulation analysis of the four resonant modes of the accelerometer.

TABLE 2: The frequencies of the accelerometer's resonant modes.

\begin{tabular}{lcccc}
\hline Resonant mode & 1 & 2 & 3 & 4 \\
\hline Resonant frequency $(\mathrm{kHz})$ & 408 & 667 & 671 & 1119 \\
\hline
\end{tabular}

after VMD is shown in Table 3; when the number of decomposed layers is set to 8 , the center frequencies of the fifth and sixth mode functions are, respectively, $536.6 \mathrm{kHz}$ and $554 \mathrm{kHz}$, which are close to each other, and mode aliasing may occur. Therefore, it is appropriate to choose 7 as the number of decomposition patterns.

To visually highlight the advantages of the VMD, VMD and EMD are compared here; Figures 8 and 9 are the decomposition results of dynamic signals obtained by using VMD and EMD, respectively. The results show that VMD can overcome the phenomenon of mode mixing and pseudocomponents caused by EMD. So, in this article, the VMD is adopted to decompose the dynamic signals.

The results are given in Figure 8; $S(t)$ is a dynamic signal which needs to be decomposed, and $S(t)$ is decomposed into
IMF components of 7 different modes. According to the second step of the VMD-PE-wavelet denoising algorithm, calculate the PE value of the acquired IMF components and then classify them into noise components, mixed components, and useful components according to the similarity. As shown in Figure 10, the range of PE values is $[0.2,0.8]$. IMF components (IMF1, IMF3, and IMF4) with PE values between 0.2 and 0.4 are determined as useful components, and these IMFs are directly reserved to participate in subsequent signal reconstruction. However, IMFs (IMF2, IMF5, and IMF6) between 0.4 and 0.6 are considered as mixed components, which need to be denoised by wavelet threshold before signal reconstruction. IMF (IMF7) with PE value over 0.6 is directly discarded as a noise component. Before signal reconstruction, we need to use the wavelet threshold to denoise the mixed components. Through a lot of comparative analysis, in this paper, wavelet basis function finally adopts the "db4" wavelet, and then the decomposition scale is set to 4. In addition, the soft threshold function is used for dealing with those wavelet coefficients. Finally, the final denoising signal can be obtained by reconstructing the IMF 

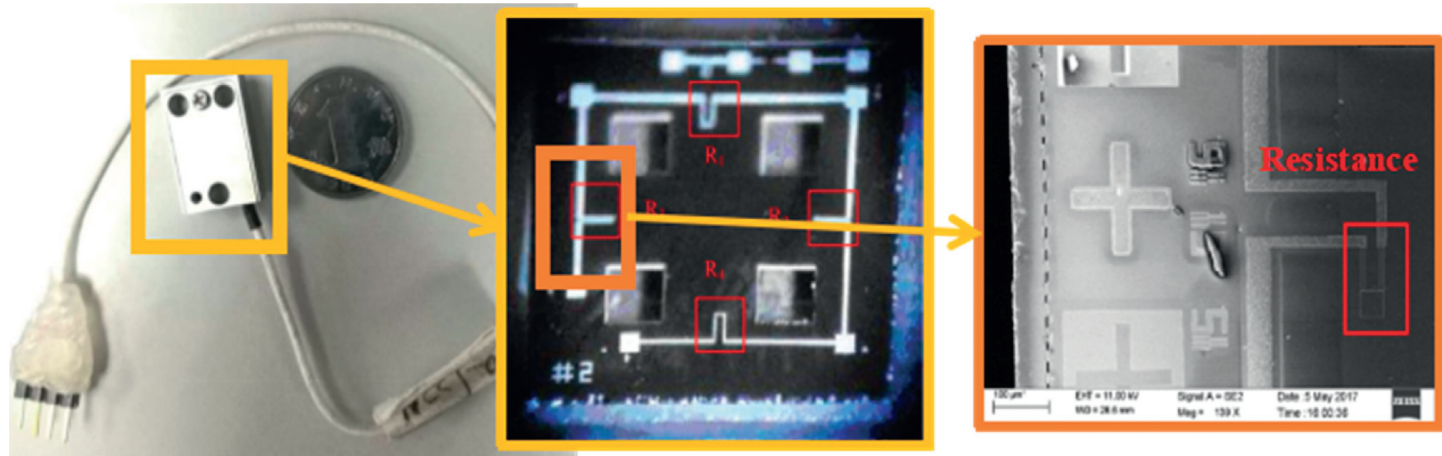

FIGURE 5: Overall picture, CCD picture, and SEM picture of the accelerometer.
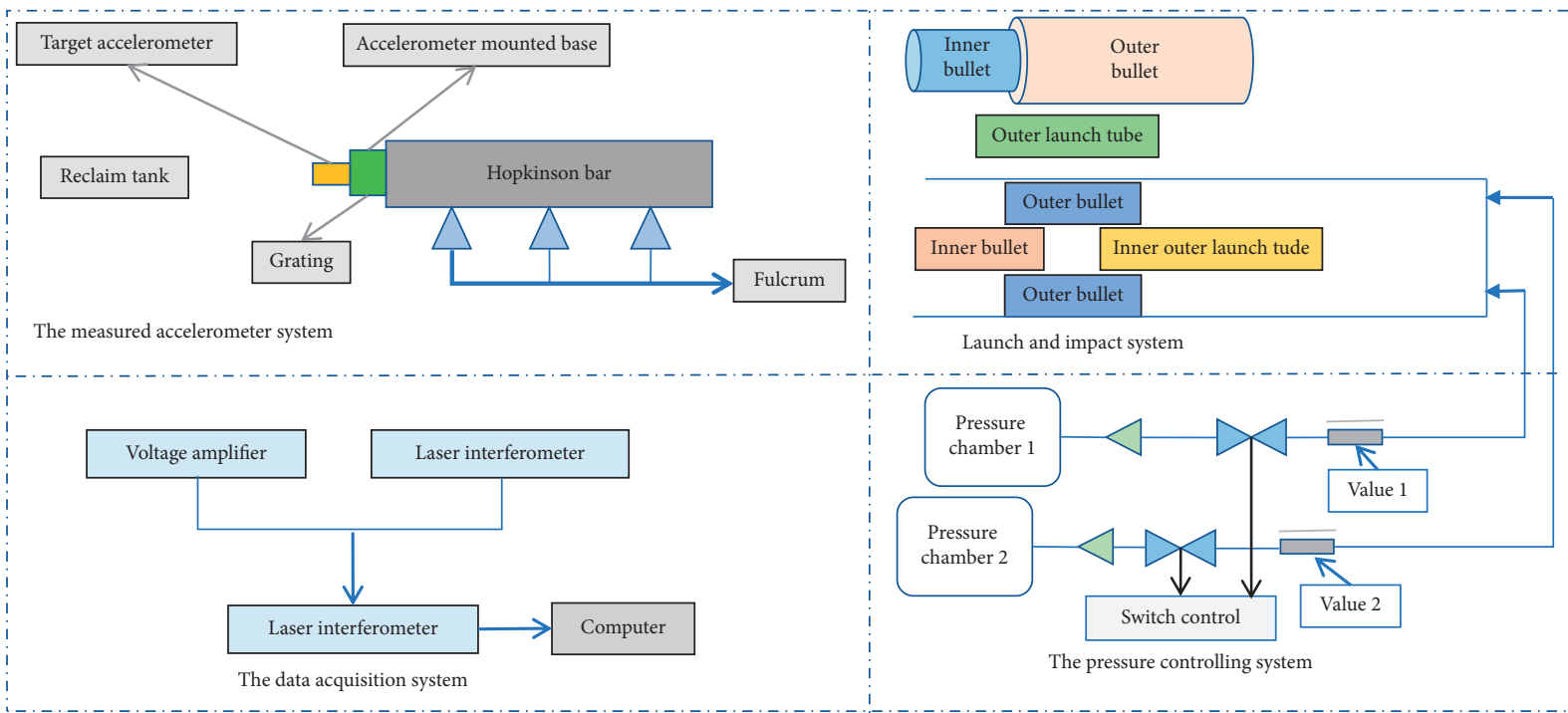

(a)

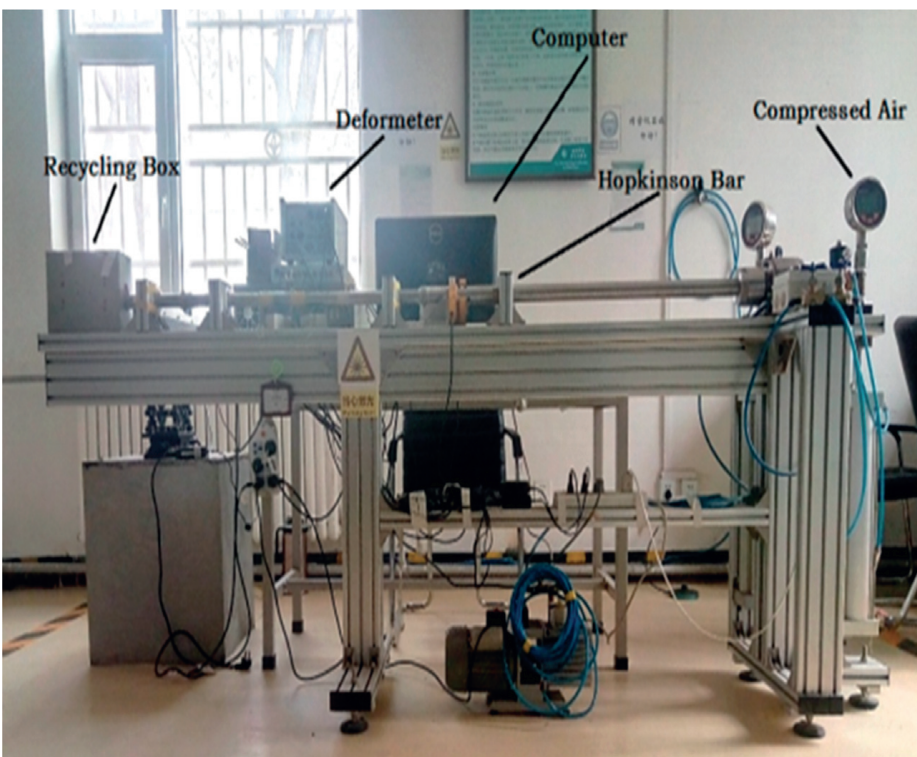

(b)

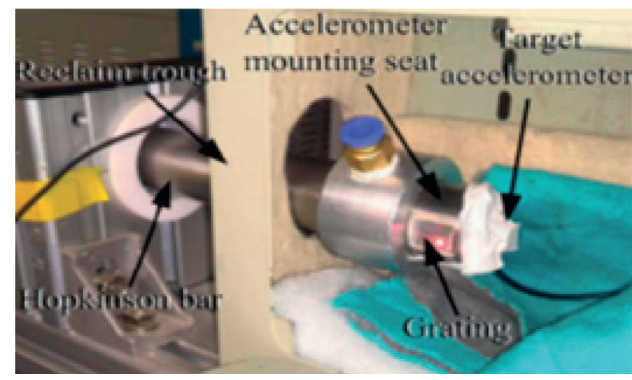

(c)

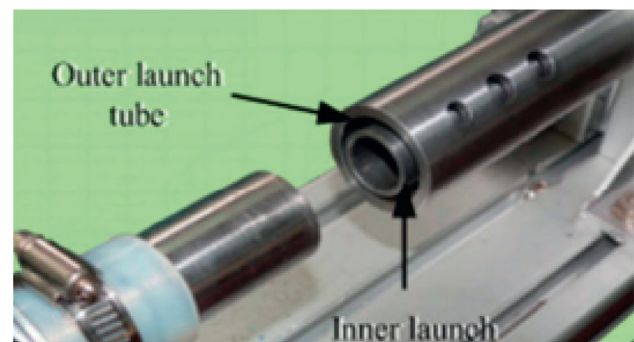

(d)

FiguRE 6: Hopkinson bar calibration device. 


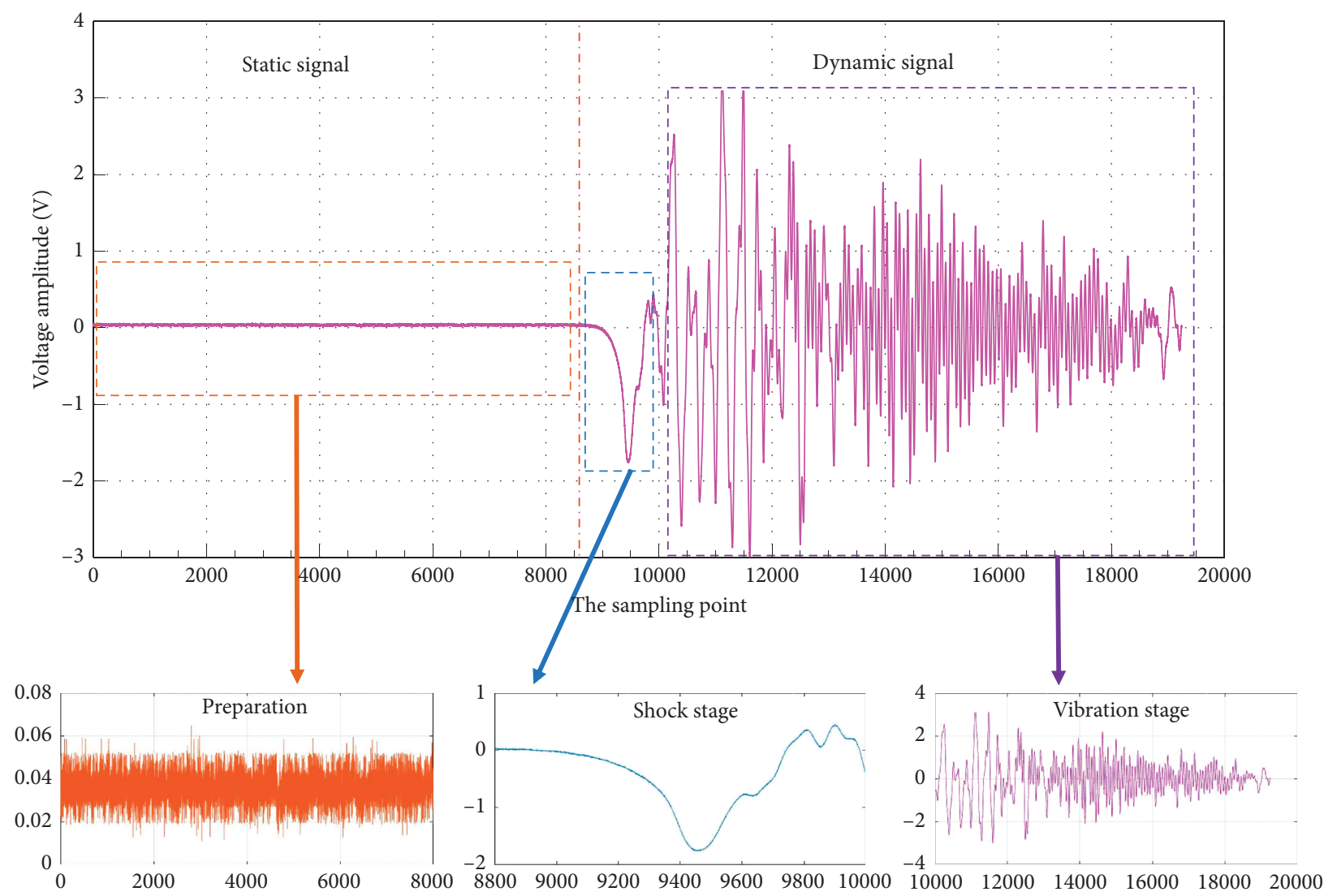

Figure 7: The output signal of the accelerometer.

TABle 3: Center frequencies of different decomposition layers.

\begin{tabular}{lcccccccc}
\hline $\begin{array}{l}\text { The value } \\
\text { of } K\end{array}$ & \multicolumn{1}{c}{ Center frequency $(\mathrm{kHz})$} \\
\hline 5 & 60.4 & 124.7 & 227 & 536.6 & 10000 & - & - & - \\
6 & 60.4 & 124.7 & 227 & 536.6 & 10000 & 10550 & - & - \\
7 & 60.4 & 124.7 & 234 & 370 & 536.6 & 10000 & 12860 & - \\
8 & 60.4 & 124.7 & 234 & 370 & 536.6 & 554 & 10000 & 12860 \\
\hline
\end{tabular}

components which have been denoised by the wavelet threshold and useful IMF components.

To verify the reliability of this new algorithm, we compared this new algorithm with EMD and wavelet threshold. The performances of different denoising methods are, respectively, discussed in both time and frequency domains. Next, the performance of different denoising algorithms will be analyzed from the preparation stage, the shock stage, and the vibration stage, respectively.

4.2.1. Preparation Stage. When there is no external interference, the output of the accelerometer is static signal. This stage contains a large number of random noises and bias characteristics of the accelerometer. It can be seen from Figure 11 that the noises in this stage are large (peak-peak is around $0.054 \mathrm{v}$ ), and the signals denoised by VMD-PEwavelet, wavelet, EMD, and VMD, respectively, are basically overlapped. In this stage, these denoising methods all have good denoising effects.

Allan variance is a time-domain analysis technique, which is often used for random error analysis of data collected by gyroscope under static conditions. Here, the Allan variance curve is used to analyze whether the noise level is reduced in the static stage (preparation stage). In Figure 12, the values of static output signals and the signals denoised by VMD-PE-wavelet threshold in $2.5 * 10^{-8} \mathrm{~s}$ are $102 \mathrm{mV} / \mathrm{h}$ and $0.65 \mathrm{mV} / \mathrm{h}$, respectively. When using VMD-PE-wavelet threshold algorithm, the noises in the original signals can be reduced by $99.97 \%$, indicating that using the VMD-PEwavelet method can significantly remove the random noise of the signals.

4.2.2. Shock Stage. For this calibration experiment, the stage is the main part, and its peak value is about $-1.754 \mathrm{v}$, and its impact pulse width is about 6.5 us. As shown in Figure 13, at this stage, the original signals, VMD-PE-wavelet threshold, and EMD denoising signals almost overlap, which indicates that these two methods can maintain useful information while removing noise. However, after the wavelet threshold denoising and VMD denoising, the signal amplitudes are only $-1.5 \mathrm{v}$ and $-1.04 \mathrm{v}$ respectively, which loses a considerable part of useful signals, and the errors are more than $14 \%$ and $40 \%$ compared to the original signals, respectively; due to serious signal distortion, wavelet threshold denoising 

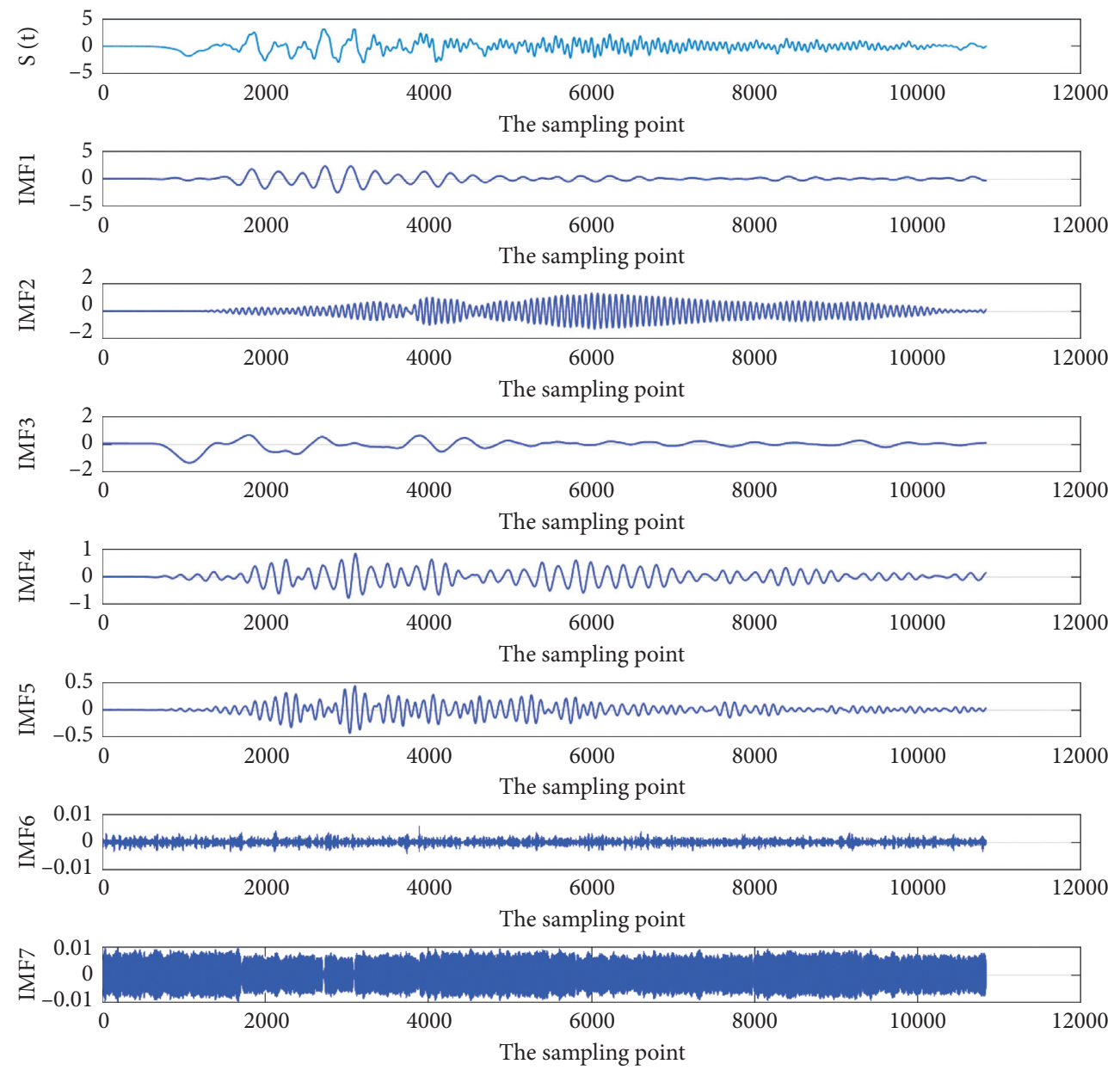

Figure 8: The decomposition results of dynamic signal by VMD.

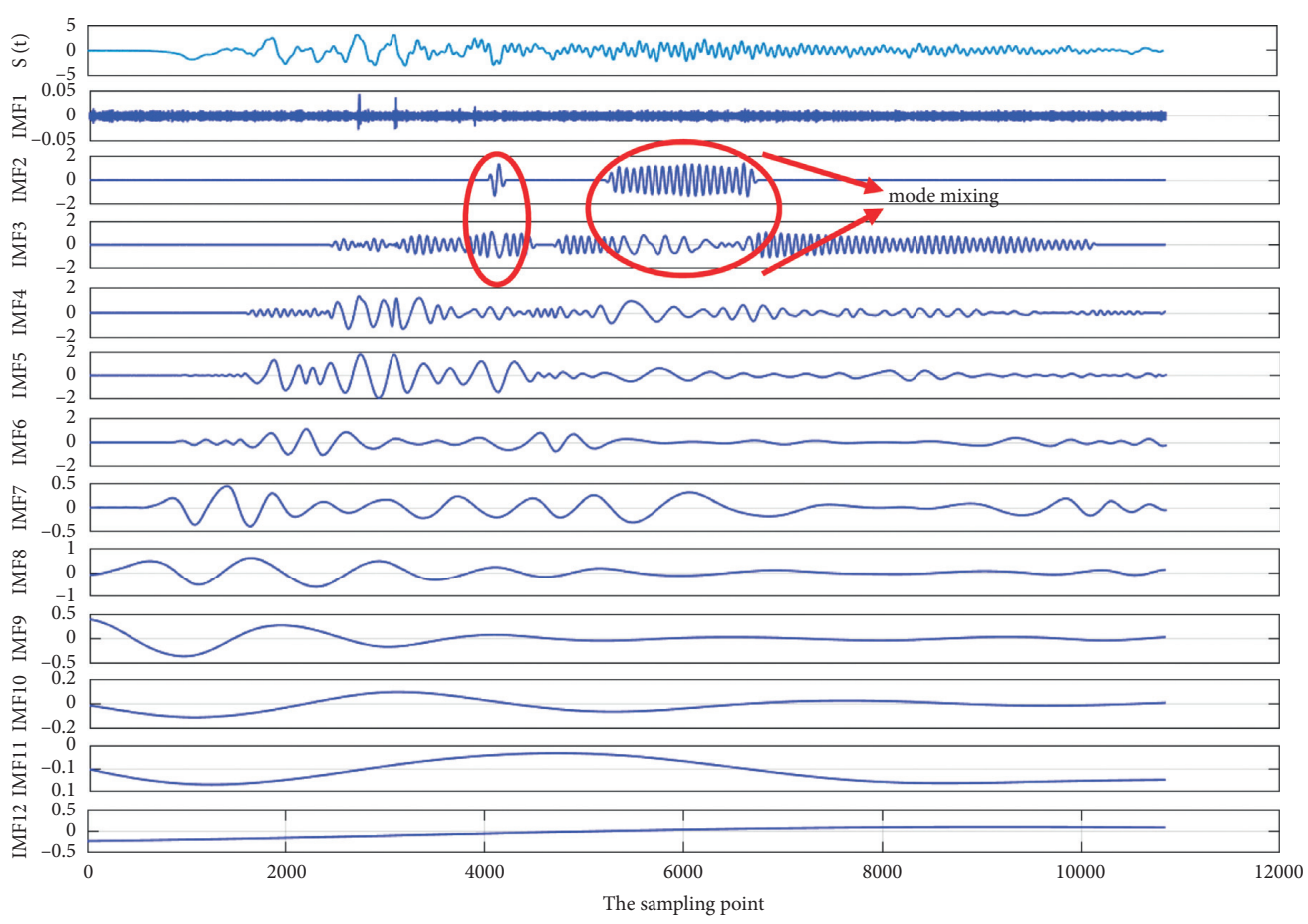

Figure 9: The decomposition results of dynamic signal by EMD. 


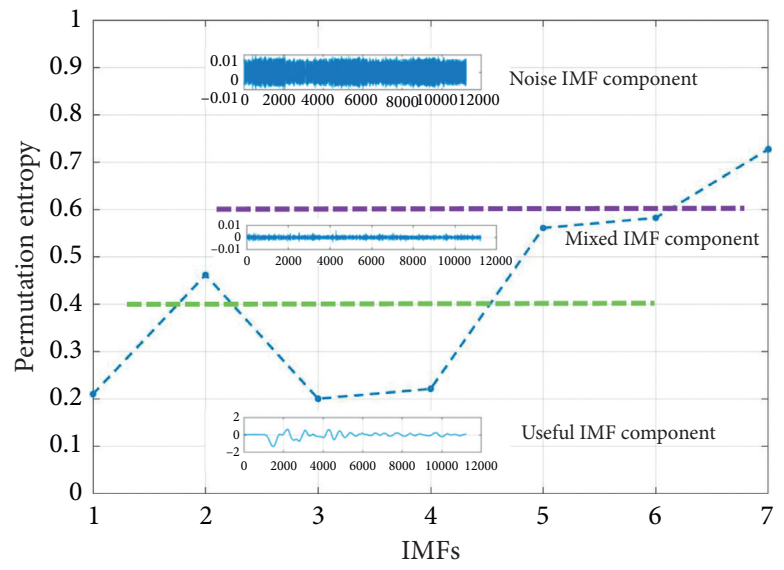

FIGURE 10: Classification of IMF components according to PE values.

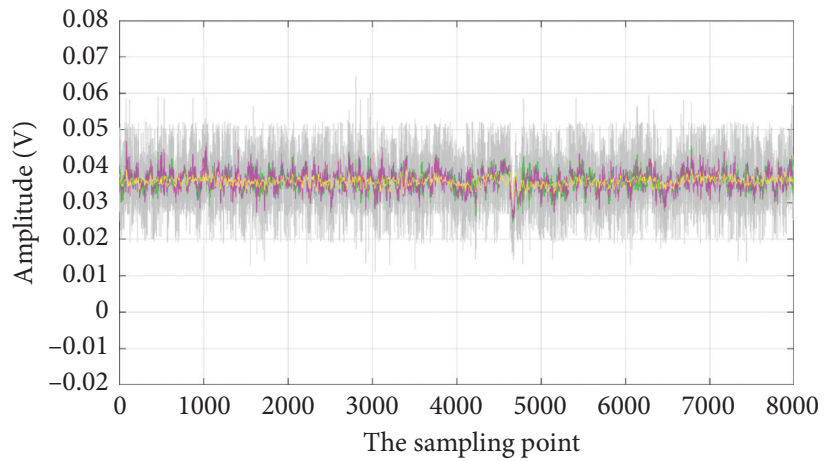

Static signal - EMD

— Wavelet threshold — VMD

— VMD-PE-wavelet threshold

FIGURE 11: Static signal denoising results.

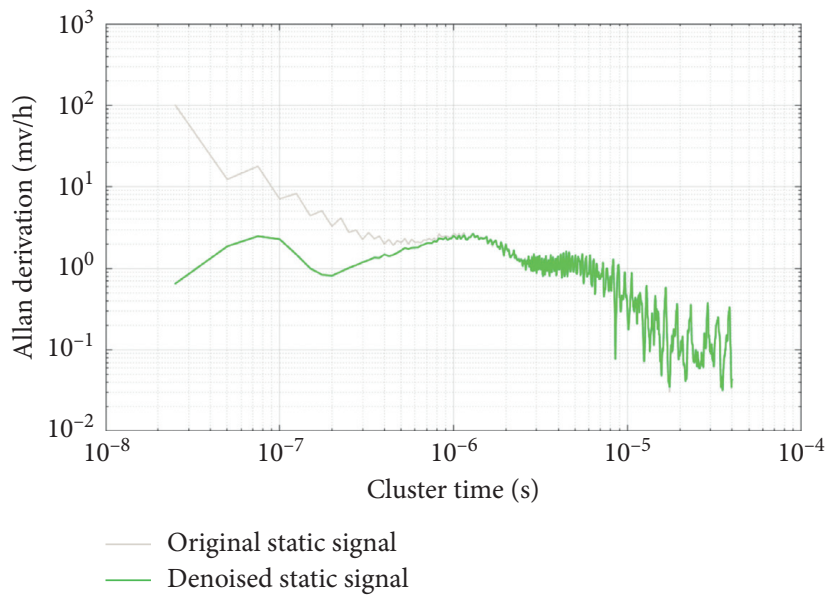

FIGURE 12: The denoising results of the preparation stage analyzed by Allan derivation. 


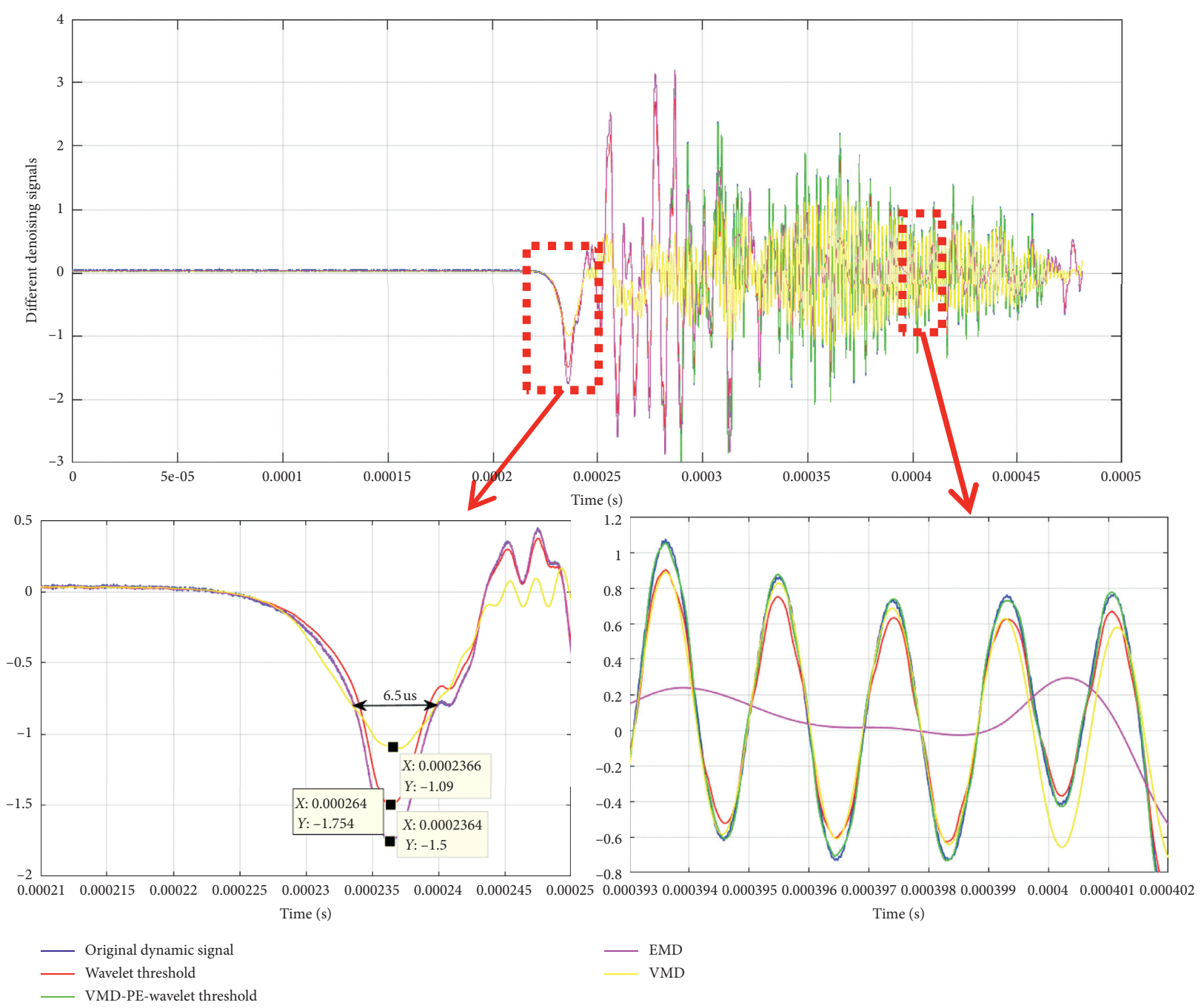

FIGURE 13: The denoising effect of different methods in dynamic stage.

and VMD algorithms are not suitable for accelerometer denoising.

4.2.3. Vibration Stage. This stage mainly contains the vibration information of the signal output by the accelerometer, and this stage reflects the dynamic characteristics of the accelerometer. The performance of different denoising algorithms at this stage is shown in Figure 13; at this stage, only VMD-PE-wavelet threshold can inherit the features of the original signal while denoising. Meanwhile, wavelet threshold denoising and VMD result in certain signal distortions, with the maximum error exceeding $17 \%$ and $25 \%$, respectively. The EMD denoising method almost loses the characteristics of the original signal; only the VMD-wavelet denoising method is applicable in the vibration phase, and the other three methods will cause serious signal distortion.

After analyzing the denoising effects of these denoising methods in time domain, we continue to discuss the frequency features of the signals after denoising with different denoising algorithms. The frequency characteristics of these signals are given in Figure 14, and the specific comparison results are summarized in Table 4.

4.2.4. Frequency Domain Analysis of the Shock Stage. At this stage, the frequency peak is around $33.2 \mathrm{kHz}$. In Figure 14, the amplitude of the signal denoised by the VMD-PEwavelet threshold denoising method has the same amplitude as the original signal at the peak point (both at $0.088 \mathrm{~V}$ ). In addition, the peak value of the signal denoised by wavelet threshold is $0.066 \mathrm{~V}$, the peak value of the signal denoised by EMD denoising is $0.045 \mathrm{~V}$, and the peak value of the signal denoised by VMD denoising is $0.060 \mathrm{~V}$; the errors are $25 \%$, $48.8 \%$, and $31.8 \%$, respectively, compared with the original signal. This means that only the VMD-PE-wavelet threshold denoising method can retain the original frequency characteristics and the actual amplitude while denoising.

4.2.5. Frequency Domain Analysis of the Vibration Stage. In the frequency domain, the frequency peak of this stage is at $536.3 \mathrm{kHz}$. In addition, the accelerometer vibrates around 

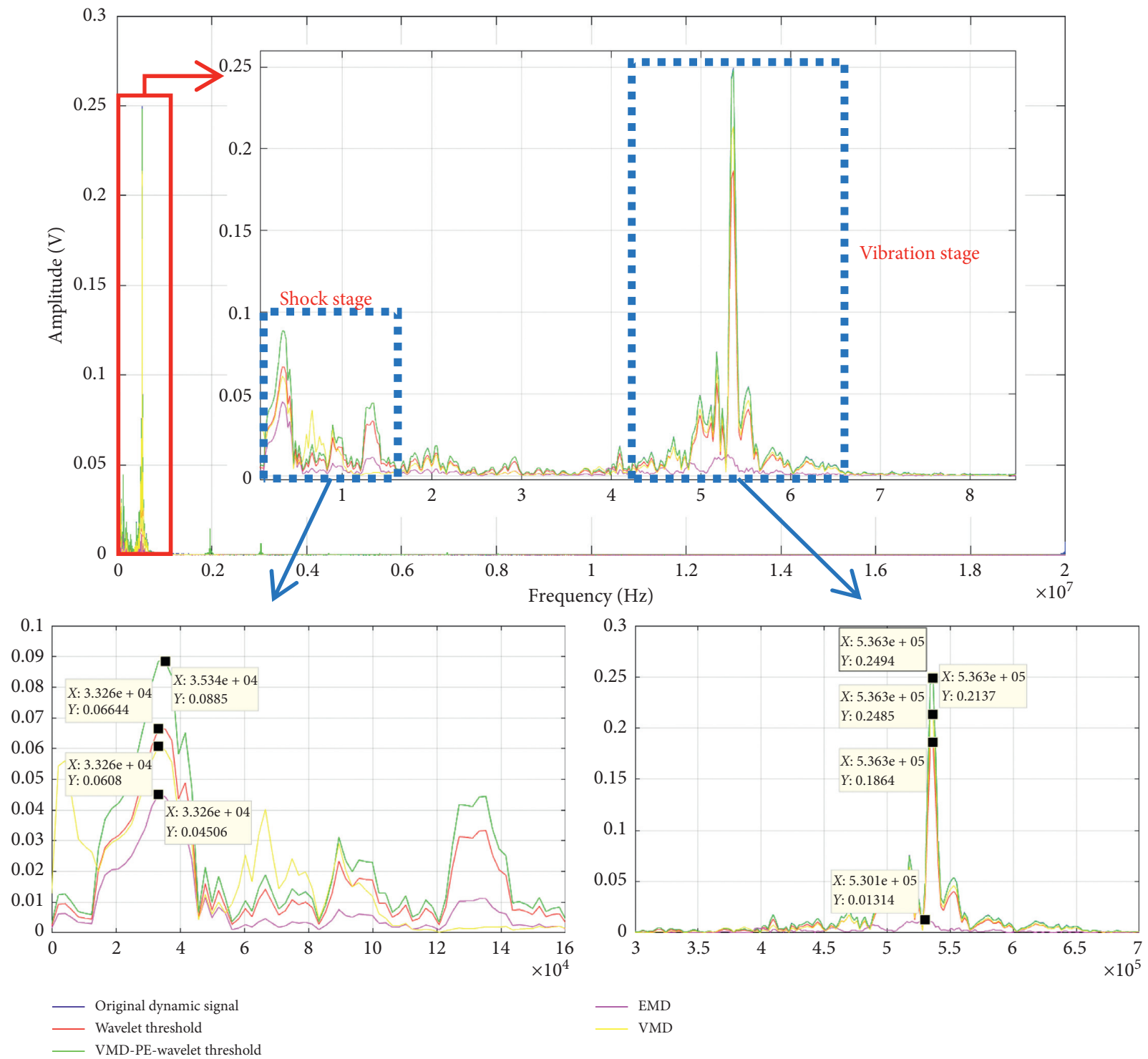

- EMD

FIGURE 14: Frequency characteristics of signals after denoising with different denoising methods.

TABLE 4: Performance evaluation of different denoising algorithms in dynamic processing.

\begin{tabular}{|c|c|c|c|c|c|c|}
\hline \multicolumn{2}{|c|}{ Methods } & Original signal & VMD-PE-wavelet & EMD & Wavelet & VMD \\
\hline \multirow{2}{*}{ Shock stage } & Value (V) & 0.088 & 0.085 & 0.066 & 0.045 & 0.060 \\
\hline & Errors (\%) & - & 3.4 & 25 & 48.8 & 31.8 \\
\hline \multirow{2}{*}{ Vibration stage } & Value (V) & 0.249 & 0.248 & 0.186 & 0.013 & 0.213 \\
\hline & Errors (\%) & - & 0.4 & 25.3 & 94.7 & 14.4 \\
\hline
\end{tabular}

the Z-axis, so just the working mode is activated. As mentioned above, the vibration frequency of the first mode of the accelerometer is $408 \mathrm{kHz}$. Due to machining errors and other factors, the actual value is deviated by about $128 \mathrm{kHz}$. As shown in Figure 14, the amplitude of the signal denoised by VMD-PE-wavelet denoising at the peak point is the same as that of the original signal (both at $0.249 \mathrm{~V}$ ). In addition, the peak value of the signal denoised by wavelet threshold denoising is $0.186 \mathrm{v}$, the peak value of the signal denoised by EMD denoising is $0.013 \mathrm{v}$, and the peak value of the signal denoised by VMD denoising is $0.213 \mathrm{v}$. Compared with the original signal, the errors are $25.3 \%, 94.7 \%$, and $14.4 \%$, respectively. These three methods lose the frequency characteristics of the original signal seriously, so only the VMD-PE-wavelet threshold denoising method can be used for accelerometer calibration denoising.

As can be seen from the above comparative analysis, VMD-PE-wavelet threshold algorithm can maintain a good signal fidelity when denoising; compared with EMD, VMD, and wavelet threshold, the signal distortion caused by 
TABle 5: Performance comparison of different denoising methods.

\begin{tabular}{lcc}
\hline Methods & SNR & Correlation coefficient \\
\hline VMD-PE-wavelet threshold & 18.56 & 0.982 \\
Wavelet threshold & 16.42 & 0.842 \\
EMD & 17.25 & 0.567 \\
VMD & 17.86 & 0.677 \\
\hline
\end{tabular}

VMD-PE-wavelet threshold algorithm is small. To compare the denoising effects of these three denoising algorithms, the correlation coefficient and signal-to-noise ratio (SNR) are introduced to quantitatively verify the superiority of this algorithm; the results are given in Table 5; the correlation coefficient and signal-to-noise ratio are defined as follows:

$$
\rho_{s_{1} s_{2}}=\frac{\operatorname{Cov}\left(S_{1}, S_{2}\right)}{\sqrt{D\left(S_{1}\right)} * \sqrt{D\left(S_{2}\right)}},
$$

where the original signal and the denoising signal are denoised as $S_{1}$ and $S_{2}, \operatorname{Cov}\left(S_{1}, S_{2}\right)$ is the covariance of $S_{1}$ and $S_{2}$, and the variance of $S_{1}$ and $S_{2}$ is denoised as $D\left(S_{1}\right)$ and $D$ $\left(S_{2}\right)$, respectively.

$$
S N R=10 \lg \left(\frac{P_{s}}{P_{n}}\right),
$$

where the effective power of the signal and noise is denoised as $P_{s}$ and $P_{n}$, respectively.

Table 5 shows the comparison results of signals denoised by different denoising methods; the signal after VMD-PEwavelet threshold denoising has the highest SNR (18.56), and its correlation coefficient is as high as 0.982 , indicating that this denoising method can maintain good signal fidelity. Compared with the other three methods, this method not only improves the SNR the most but also reduces the signal distortion.

\section{Conclusions}

In order to eliminate the noises in the accelerometer output signals to achieve the purpose of calibration denoising, this article proposes a joint denoising algorithm which combines the wavelet threshold with the VMD and PE algorithms. Compared with the current decomposition algorithm (EMD, LMD, etc.), VMD-PE has the advantages of antimode aliasing and good mode recognition ability. The core idea of this method is firstly, the output signals are decomposed and classified by VMD-PE, and then the mixed component is processed by the wavelet threshold, which avoids the signal distortion caused by the direct use of wavelet threshold denoising. This combined denoising method combines the decomposition ability of VMD, the recognition ability of PE, and the denoising ability of wavelet threshold, which can achieve a balance between denoising effect and signal fidelity. The experiment shows that this method not only has a good denoising effect (the noises in the static part can be eliminated by $99.97 \%$ and the SNR of the dynamic part is raised to 18.56) but also can maintain a good signal fidelity (the error of shock peak amplitude is 3.4\%, the error of vibration peak amplitude is $0.4 \%$, and the correlation coefficient between the denoising signal and the dynamic part is as high as 0.982). Therefore, the combined denoising algorithm (VMD-PE-wavelet threshold) proposed in this article has good denoising effect and is suitable for accelerometer calibration.

\section{Data Availability}

The data used to support the findings of this study are available from the corresponding author upon request.

\section{Conflicts of Interest}

The authors declare that they have no conflicts interest.

\section{Authors' Contributions}

$\mathrm{HC}$ and $\mathrm{ZZ}$ contributed equally to this paper. $\mathrm{HC}, \mathrm{ZZ}$, and $\mathrm{RZ}$ conceived and designed the experiments. HC and YS performed the experiments. ZZ and XC analyzed the data. ZZ wrote the paper.

\section{Acknowledgments}

This study was supported by the National Natural Science Foundation of China (nos. 51705477 and 61973281), the Aeronautical Science Foundation of China (2019080U0002), the Key Research and Development (R\&D) Projects of Shanxi Province (201903D111005), and the Fund for Shanxi "1331 Project" Key Subjects Construction.

\section{References}

[1] A. G. Krause, M. Winger, T. D. Blasius, Q. Lin, and O. Painter, "A high-resolution microchip optomechanical accelerometer," Nature Photonics, vol. 6, no. 11, p. 768, 2012.

[2] V. Narasimhan, H. Li, and M. Jianmin, "Micromachined high-g accelerometers: a review," Journal of Micromechanics and Microengineering, vol. 25, no. 3, Article ID 33001, 2015.

[3] W. Xu, J. Yang, G. Xie et al., "Design and fabrication of a slanted-beam MEMS accelerometer," Micromachines, vol. 8, no. 3, p. 77, 2017.

[4] H. Cao, Y. Zhang, Z. Han et al., "Pole-zero temperature compensation circuit design and experiment for dual-mass MEMS gyroscope bandwidth expansion," IEEE/ASME Transactions on Mechatronics, vol. 24, no. 2, pp. 677-688, 2019.

[5] C. Shen, Y. Zhang, J. Tang, H. Cao, and J. Liu, "Dual-optimization for a MEMS-INS/GPS system during GPS outages based on the cubature Kalman filter and neural networks," Mechanical Systems and Signal Processing, vol. 133, Article ID 106222, 2019.

[6] H. Cao, Y. Liu, Z. Kou et al., "Design, fabrication and experiment of double U-beam MEMS vibration ring gyroscope," Micromachines, vol. 10, no. 3, p. 186, 2019.

[7] H. Cao, H. Li, J. Liu, Y. Shi, J. Tang, and C. Shen, "An improved interface and noise analysis of a turning fork microgyroscope structure," Mechanical Systems and Signal Processing, vol. 70-71, pp. 1209-1220, 2016. 
[8] Y. Shi, Y. Zhao, H. Feng et al., "Design, fabrication and calibration of a high-G MEMS accelerometer," Sensors and Actuators A: Physical, vol. 279, pp. 733-742, 2018.

[9] D. L. Donoho and I. M. Johnstone, "Adapting to unknown smoothness via wavelet shrinkage," Journal of the American Statistical Association, vol. 90, no. 432, pp. 1200-1224, 1995.

[10] D. L. Donoho and I. M. Johnstone, "Ideal spatial adaptation via wavelet shrinkage," Biometrika, vol. 81, no. 12, pp. 425-455, 1994.

[11] J. S. Smith, "The local mean decomposition and its application to EEG perception data," Journal of the Royal Society Interface, vol. 2, no. 5, pp. 443-454, 2005.

[12] K. Dragomiretskiy and D. Zosso, "Variational mode decomposition," IEEE Transactions on Signal Processing, vol. 62, no. 3, pp. 531-544, 2013.

[13] Z. Li, J. Chen, Y. Zi, and J. Pan, "Independence-oriented VMD to identify fault feature for wheel set bearing fault diagnosis of high speed locomotive," Mechanical Systems and Signal Processing, vol. 85, pp. 512-529, 2017.

[14] M. Zhang, Z. Jiang, and K. Feng, "Research on variational mode decomposition in rolling bearings fault diagnosis of the multistage centrifugal pump," Mechanical Systems and Signal Processing, vol. 93, pp. 460-493, 2017.

[15] Y. Wang, R. Markert, J. Xiang, and W. Zheng, "Research on variational mode decomposition and its application in detecting rub-impact fault of the rotor system," Mechanical Systems and Signal Processing, vol. 60-61, pp. 243-251, 2015.

[16] C. Shen, J. Yang, J. Tang, J. Liu, and H. Cao, "Note: parallel processing algorithm of temperature and noise error for micro-electro-mechanical system gyroscope based on variational mode decomposition and augmented nonlinear differentiator," Review of Scientific Instruments, vol. 89, no. 7, Article ID 76107, 2018.

[17] R. Sharma, R. Pachori, and U. Acharya, "Application of entropy measures on intrinsic mode functions for the automated identification of focal electroencephalogram signals," Entropy, vol. 17, no. 2, pp. 669-691, 2015.

[18] L. Meng, J. Xiang, Y. Wang, Y. Jiang, and H. Gao, “A hybrid fault diagnosis method using morphological filter-translation invariant wavelet and improved ensemble empirical mode decomposition," Mechanical Systems and Signal Processing, vol. 50-51, pp. 101-115, 2015.

[19] Z. Liu, Z. He, W. Guo, and Z. Tang, "A hybrid fault diagnosis method based on second generation wavelet de-noising and local mean decomposition for rotating machinery," ISA Transactions, vol. 61, pp. 211-220, 2016.

[20] S. Lahmiri, "Comparative study of ECG signal denoising by wavelet thresholding in empirical and variational mode decomposition domains," Healthcare Technology Letters, vol. 1, no. 3, pp. 104-109, 2014.

[21] Z.-X. Yang and J.-H. Zhong, "A hybrid EEMD-based SampEn and SVD for acoustic signal processing and fault diagnosis," Entropy, vol. 18, no. 4, p. 112, 2016.

[22] X. Wang, J. Meng, Y. Xu, Z. Chen, and J. Luo, "ECG compression based on combining of EMD and wavelet transform," Electronics Letters, vol. 52, no. 19, pp. 1588-1590, 2016.

[23] F. Bi, T. Ma, and X. Wang, "Development of a novel knock characteristic detection method for gasoline engines based on wavelet-denoising and EMD decomposition," Mechanical Systems and Signal Processing, vol. 117, pp. 517-536, 2019.

[24] L. Bai, Z. Han, Y. Li, and S. Ning, "A hybrid de-noising algorithm for the gear transmission system based on CEEMDAN-PE-TFPF," Entropy, vol. 20, no. 5, p. 361, 2018.
[25] C. Bandt and B. Pompe, "Permutation entropy: a natural complexity measure for time series," Physical Review Letters, vol. 88, no. 17, Article ID 174102, 2002.

[26] X. Zhang, Y. Liang, J. Zhou, and Y. Zang, "A novel bearing fault diagnosis model integrated permutation entropy, ensemble empirical mode decomposition and optimized SVM," Measurement, vol. 69, pp. 164-179, 2015.

[27] C. Yi, Y. Lv, M. Ge, H. Xiao, and X. Yu, “Tensor singular spectrum decomposition algorithm based on permutation entropy for rolling bearing fault diagnosis," Entropy, vol. 19, no. 4, p. 139, 2017.

[28] L. Zhao, W. Yu, and R. Yan, "Gearbox fault diagnosis using complementary ensemble empirical mode decomposition and permutation entropy," Shock and Vibration, vol. 20168 pages, 2016.

[29] Z. Shi, W. Song, and S. Taheri, "Improved LMD, permutation entropy and optimized K-means to fault diagnosis for roller bearings," Entropy, vol. 18, no. 3, p. 70, 2016.

[30] Z. Wang, J. Wang, W. Cai et al., "Application of an improved ensemble local mean decomposition method for gearbox composite fault diagnosis," Complexity, vol. 2019, Article ID 1564243, 17 pages, 2019.

[31] Y. Yang, M. Zhou, Y. Niu et al., "Epileptic seizure prediction based on permutation entropy," Frontiers in Computational Neuroscience, vol. 12, p. 55, 2018.

[32] M. Schweigmann, K. P. Koch, F. Auler, and F. Kirchhoff, "Improving electrocorticograms of awake and anaesthetized mice using wavelet denoising," Current Directions in Biomedical Engineering, vol. 4, no. 1, pp. 469-472, 2018.

[33] X. Zhang, "A modified artificial bee colony algorithm for image denoising using parametric wavelet thresholding method," Pattern Recognition and Image Analysis, vol. 28, no. 3, pp. 557-568, 2018.

[34] B. Yu, S. Li, C. Chen et al., "Prediction subcellular localization of Gram-negative bacterial proteins by support vector machine using wavelet denoising and Chou's pseudo amino acid composition," Chemometrics and Intelligent Laboratory Systems, vol. 167, pp. 102-112, 2017. 